\title{
Erratum: Measure of nonlocality which is maximal for maximally entangled qutrits [Phys. Rev. A 92, 030101(R) (2015)]
}

\section{E. A. Fonseca and Fernando Parisio}

(Received 25 February 2016; published 9 March 2016)

DOI: 10.1103/PhysRevA.93.039904

There is a missing term in Eq. (4). It should read

$$
\begin{aligned}
I_{3}= & P\left(A_{1}=B_{1}\right)+P\left(B_{1}=A_{2}+1\right)+P\left(A_{2}=B_{2}\right)+P\left(B_{2}=A_{1}\right) \\
& -P\left(A_{1}=B_{1}-1\right)-P\left(B_{1}=A_{2}\right)-P\left(B_{2}=A_{1}-1\right)-P\left(A_{2}=B_{2}-1\right) \leqslant 2 .
\end{aligned}
$$

There is a wrong sign in Eq. (6) and the normalizing factor is missing. Its correct form is

$$
\left|\Psi^{\prime}\right\rangle=\frac{1}{3 \sqrt{2+\gamma^{2}}} \sum_{j, k, l=0}^{2} \alpha_{j} e^{i\left[\phi_{a}(j)+\varphi_{b}(j)\right]} e^{i \frac{2 \pi}{3} j(k-l)}|k l\rangle .
$$

To obtain all the results presented in the article, we have employed the correct equations. 